\title{
THREE THEOREMS APPLICABLE TO VIBRATION THEORY*
}

BY B. F. KIMBALL

1. Introduction. In discussions of the theory of free vibrations it is sometimes stated without proof that "the fact that the period of vibration is independent of the amplitude shows that the restoring force is linear." The present paper includes a proof of this statement and a brief consideration of the case of a nonlinear restoring force.

2. Hypotheses and Existence of Period Integral. (A) Let the restoring force $f(x)$ be a continuous function of the distance $x$ from the position of equilibrium on the interval

$$
0 \leqq x \leqq D \text {. }
$$

Let $f^{\prime}(x)$ exist on this interval. Let $f(0)=0$ and

$$
f(x)>0, f^{\prime}(x)>0 \text { on } 0<x \leqq D .
$$

We suppose also that $f(x)$ is symmetric in $x=0$; that is, we define the function over the interval $-D \leqq x \leqq 0$ so that

$$
f(+x)=-f(-x) .
$$

(B) Take the derivative

$$
f^{\prime}(0)=b>0 .
$$

(C) Let the unilateral derivative $f^{\prime \prime}\left(0^{+}\right)$exist.

The differential equation of vibration is

$$
\frac{d^{2} x}{d t^{2}}=-f(x)
$$

and one obtains as an integral

$$
t=\int_{0}^{x}(C-2 F(x))^{-1 / 2} d x+\text { const. }
$$

where $F(x)=\int_{0}^{x} f(x) d x$ and $C$ is a constant of integration. Let $a$

* Presented to the Society, March 26, 1932. 
denote the maximum displacement from the position of equilibrium where $a$ lies on interval (1). This we call the amplitude of the vibration. Then the integral which applies to the motion is

$$
t=2^{-1 / 2} \int_{0}^{x}(F(a)-F(x))^{-1 / 2} d x .
$$

Thus the period of vibration $T$ is given by

$$
T=2 \cdot 2^{1 / 2} \int_{0}^{a}(F(a)-F(x))^{-1 / 2} d x
$$

if this integral exists.

The existence of the integral can be established as follows. Set

$$
Q(x, a)=(F(a)-F(x)) /(a-x),
$$

and the integral becomes

$$
T=2 \cdot 2^{1 / 2} \int_{0}^{a}(a-x)^{-1 / 2}(Q(x, a))^{-1 / 2} d x .
$$

Now $Q(x, a)$ is continuous in $x$ except at $x=a$. Furthermore

We define

$$
\lim _{x \rightarrow a} Q(x, a)=F^{\prime}(a) \text {. }
$$

$$
Q(a, a)=F^{\prime}(a)
$$

and note that

$$
Q(0, a)=F(a) / a .
$$

By definition $F(x)<F(a)$ for $x<a$. Hence one concludes that $Q(x, a)$ is a positive continuous function on the interval $0 \leqq x \leqq a$ and the existence of integral (4) follows.

3. Double Inequality Satisfied by Period. We wish to establish a double inequality bounding $T$. Apply the first law of the mean for integrals to (4). This gives

$$
T=2 \cdot 2^{1 / 2}(Q(\bar{x}, a))^{-1 / 2} \int_{0}^{a}(a-x)^{-1 / 2} d x=4 \cdot 2^{1 / 2}(a / Q(\bar{x}, a))^{1 / 2} .
$$

Now it can be shown that $Q^{\prime}(x, a)$ is positive on the interval 
$0 \leqq x<a$. In order to show this note that

$$
Q^{\prime}(x, a)=\frac{Q(x, a)-F^{\prime}(x)}{a-x} .
$$

Apply the law of the mean to $Q(x, a)$ and we have

$$
Q^{\prime}(x, a)=\left(F^{\prime}(u)-F^{\prime}(x)\right) /(a-x), x<u<a .
$$

Since $f^{\prime}(x)$ is positive, $F^{\prime}(x)$ is an increasing function of $x$ and thus $Q^{\prime}(x, a)$ is positive on $0 \leqq x \leqq a$. Hence referring to $(5)$ and (6) we have

$$
F(a) / a<Q(\bar{x}, a)<F^{\prime}(a),
$$

and $T$ must satisfy the relation

$$
4 \cdot 2^{1 / 2}\left(a / F^{\prime}(a)\right)^{1 / 2}<T<4 \cdot 2^{1 / 2}\left(a^{2} / F(a)\right)^{1 / 2},
$$

or, since $F^{\prime}(x)=f(x)$,

$$
4 \cdot 2^{1 / 2}(a / f(a))^{1 / 2}<T<4 \cdot 2^{1 / 2}\left(a^{2} / F(a)\right)^{1 / 2} .
$$

Hence we may state the following theorem.

THEOREM 1. The period $T$ of a free vibration due to a restoring force $f(x)$ conditioned by hypothesis (A) satisfies the double inequality (9), where $a$ is the amplitude and $F(a)=\int_{0}^{a} f(x) d x$.

4. A Necessary Condition that Period be Independent of Amplitude. First we note that if $f^{\prime}(0)=0$, it follows from the inequality established above that $T \rightarrow \infty$ as $a \rightarrow 0$. On the other hand if $a \neq 0$, $T$ is finite. Hence if $f^{\prime}(0)=0, T$ is not independent of $a$.

Before going further we shall establish the following lemma for the sake of clarity.

Lemma 1. Given function $\psi(x)$, where $\psi(0)=A, \psi^{\prime}(x)=B$, $\psi^{\prime \prime}(0)=C$, and where $\psi^{\prime}(x)$ exists for $x>0, \psi^{\prime \prime}(x)$ does not necessarily exist for $x>0$; then

$$
\lim _{x \rightarrow 0}(\psi(x)-A-B x) / x^{2}=\frac{1}{2} C .
$$

In order to prove this lemma write

$$
p(x)=\psi(x)-A-B x-\frac{1}{2} C x^{2} .
$$

Then $p(0)=0, p^{\prime}(0)=0$ and $p^{\prime}(x)$ exists for $x>0, p^{\prime \prime}(0)=0$. Now 


$$
\lim _{x \rightarrow 0}(\psi(x)-A-B x) / x^{2}=\frac{1}{2} C+\lim _{x \rightarrow 0} p(x) / x^{2} .
$$

Write $p(x)=x p^{\prime}(\theta)$, where $0<\theta<x$. Then it is clear that

$$
\lim _{x \rightarrow 0} p(x) / x^{2}=\lim _{x \rightarrow 0}\left(\theta p^{\prime}(\theta)\right) /(x \theta)=0,
$$

and the lemma is established.

We now undertake to find a necessary condition that $T$ be independent of $a$ under hypotheses (A) and (B) of $\$ 2$. The quantity $b$ satisfies the relation $b=f^{\prime}(0)=F^{\prime \prime}(0)$. Consider the function $\phi(x)$ defined by the equation

$$
\phi(x)=2 F(x) /\left(b x^{2}\right), \quad 0<x \leqq D .
$$

From the above lemma

$$
\lim _{x \rightarrow 0} 2 F(x) /\left(b x^{2}\right)=1
$$

If $\phi(0)=1$ by definition, $\phi(x)$ is a continuous function of $x$ on the interval $0 \leqq x \leqq D$. Thus $\phi(x)$ takes on its absolute maximum and minimum values on this interval. Let $\phi\left(x_{0}\right)$ be the maximum and $\phi\left(x_{1}\right)$ the minimum. Denote by $T(z)$ the period of vibration due to force $f(x)$ with amplitude $z$. Comparing $\phi(x)$ and $\phi\left(x_{0}\right)$ we have

$$
F(x) / F\left(x_{0}\right) \leqq x^{2} / x_{0}^{2}, 0 \leqq x \leqq x_{0} .
$$

Hence

$$
\int_{0}^{x_{0}}\left(1-F(x) / F\left(x_{0}\right)\right)^{-1 / 2} d x \leqq \int_{0}^{x_{0}}\left(1-x^{2} / x_{0}^{2}\right)^{-1 / 2} d x .
$$

Again, since $\phi(0)=1$, we judge that $\left(F\left(x_{0}\right)\right)^{-1 / 2} \leqq\left(\frac{1}{2} b x_{0}^{2}\right)^{-1 / 2}$. Multiplying this inequality by (11) one finds that

(12) $T\left(x_{0}\right) \leqq 4 /\left(x_{0} b^{1 / 2}\right) \int_{0}^{x_{0}}\left(1-x^{2} / x_{0}^{2}\right)^{-1 / 2} d x=2 \pi b^{-1 / 2}$.

By a similar argument one finds that

$$
T\left(x_{1}\right) \geqq 2 \pi b^{-1 / 2} .
$$

Now, if $\phi\left(x_{0}\right)>1$, the inequality sign will apply to (10) for some values of $x$, and thus the inequality sign will have to te used in (12). Similarly, if $\phi\left(x_{1}\right)<1$, the inequality sign must be used 
in (13). Hence, if $T(z)$ is to be independent of $z$, we must have $\phi(x) \equiv 1$, which means that $f(x) \equiv b x$. Thus we have the following theorem.

THEOREM 2. In order that the period of a vibration produced by a force $f(x)$, conditioned by hypotheses (A) and (B), be independent of the amplitude it is necessary and sufficient that $f(x)$ be a linear function of $x$ of the form $b x$.

5. Limit Approached by Period as Amplitude Approaches Zero. In this section we employ hypotheses (A), (B) and (C) of $\$ 2$. We find it convenient to use the function $\phi(x)$ defined above. We write

and

$$
\begin{aligned}
& F(x)=\frac{1}{2} b x^{2} \phi(x), \\
& F(a)=\frac{1}{2} b a^{2} \phi(a),
\end{aligned}
$$

Now

$$
\phi(x)=\phi(a)-(a-x) \phi^{\prime}\left(u_{2}\right), x<u_{2}<a .
$$

$$
\phi^{\prime}(0)=\lim _{x \rightarrow 0}(\phi(x)-\phi(0)) / x=(2 / b) \lim _{x \rightarrow 0}\left(F(x)-\frac{1}{2} b x^{2}\right) / x^{3} .
$$

Thus using Lemma 1 extended to apply to the third derivative, we find that this limit is $F^{\prime \prime \prime}\left(0^{+}\right) /(3 b)$ and hence

$$
\phi^{\prime}\left(0^{+}\right)=f^{\prime \prime}(0) /(3 b) \text {. }
$$

Thus $\phi^{\prime}(x)$ is bounded on the interval

$$
0 \leqq x \leqq a .
$$

We write

$$
F(a)-F(x)=\frac{1}{2} b \phi(a)\left(a^{2}-x^{2}\right)+\frac{1}{2} b x^{2}(a-x) \phi^{\prime}\left(u_{2}\right),
$$

and note that from the above discussion $\lim _{a \rightarrow 0} \phi^{\prime}\left(u_{2}\right) x^{2} /(a+x)$ is equal to zero and uniformly convergent for $x$ on (15). Also $\lim _{a \rightarrow 0} \phi(a)=1$. Hence

$$
F(a)-F(x)=\frac{1}{2} b\left(a^{2}-x^{2}\right)(1+e(x, a)),
$$

where $e(x, a)$ converges uniformly to zero with $a$ for $x$ on (15). Accordingly

$$
(F(a)-F(x))^{-1 / 2}=\left(\frac{1}{2} b\left(a^{2}-x^{2}\right)\right)^{-1 / 2}(1+h(x, a)),
$$

where $h(x, a)$ converges uniformly to zero with $a$ for $x$ on (15). 
One is thus led to the conclusion that

$$
\lim _{a \rightarrow 0} 2 \cdot 2^{1 / 2} \int_{0}^{a}(F(a)-F(x))^{-1 / 2} d x=2 \pi b^{-1 / 2} .
$$

Hence we have the following theorem.

THEOREM 3. The period of vibration $T$ under restoring force $f(x)$, conditioned by hypotheses (A), (B), and (C), approaches the limit $2 \pi b^{-1 / 2}$ as the amplitude approaches zero.

Schenectady, N. Y.

\section{A NOTE ON FERMAT'S LAST THEOREM}

$$
\text { D. H. LEHMER* }
$$

In $1925 \mathrm{H}$. S. Vandiver $†$ proved the following theorem.

THEOREM 1. If

$$
x^{p}+y^{p}+z^{p}=0
$$

is satisfied by integers $x, y, z$, prime to the odd prime $p$, then the first factor of the class number of the field generated by $e^{2 \pi i / p}$ is divisible by $p^{8}$.

In the seventh of a series of articles on Fermat's last theorem, T. Morishima $\ddagger$ has given the following improvement upon Theorem 1.

Theorem 2. In Theorem 1 we may replace $p^{8}$ by $p^{12}$ provided $p$ does not divide $75571 \cdot 20579903$.

It is the purpose of this note to show that the proviso of Theorem 2 is unnecessary by showing that (1) is not satisfied by the prime factors of $75571 \cdot 20579903$. This is done by applying Wieferich's $\|$ criterion.

TheOREM 3. If (1) is satisfied by integers $x, y, z$, prime to $p$, then $2^{p-1} \equiv 1\left(\bmod p^{2}\right)$.

* National Research Fellow.

$\dagger$ Annals of Mathematics, (2), vol. 26, p. 232.

$\ddagger$ Proceedings of the Imperial Academy of Japan, vol. 8 (1932), pp. 63-66.

\| Journal für Mathematik, vol. 136 (1909), p. 203. 